\title{
Reliability and Validity of Biostatical Analysis used for Pharmacy Research Questionnaire in Saudi Arabia
}

\author{
Yousef Ahmed Alomi*, (iD Critical \\ Care Clinical Pharmacists, TPN Clinical \\ Pharmacist, Freelancer Business Planner, \\ Content Editor and Data Analyst, Riyadh, \\ Saudi Arabia. \\ Ismail Hassan Amer Abu Rayah, Health \\ Management Registrar, General Director- \\ ate of Medical Consultations, Riyadh, \\ Saudi Arabia.
}

\section{Correspondence:}

Dr. Yousef Ahmed Alomi, BSc. Pharm,

MSc. Clin Pharm, BCPS, BCNSP, DiBA, CDE, Critical care clinical pharmacists, TPN

Clinical Pharmacist, Freelancer Business Planner, Content Editor and Data Analyst, P.O.BOX 100, Riyadh 11392, Riyadh, Saudi Arabia

Phone no: +966504417712

E-mail: yalomi@gmail.com
Received: 20-07-2019;

Accepted: 10-12-2019.

Copyright: (c) the author(s),publisher and licensee Pharmacology, Toxicology and Biomedical Reports. This is an open-access article distributed under the terms of the Creative Commons Attribution NonCommercial License, which permits unrestricted non-commercial use, distribution, and reproduction in any medium, provided the original work is properly cited.

This is an open access article distributed under the terms of the Creative Commons Attribution-NonCommercial-ShareAlike 4.0 License

Access this article online

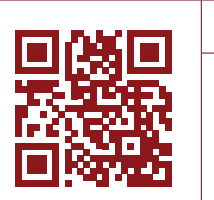

www.ptbreports.org

DOI:

10.5530/PTB.2020.6.18

\begin{abstract}
Objectives: To declare reliability and validity of Biostatical Analysis used for Pharmacy Research Survey in Saudi Arabia. Methods: It is a cross-section survey developed by the authors and the research team. It's based on the updated literature, national and international accreditation standards organizations. The Internal consistency, reliability through inter-rater reliability, item-item coloration, item-total coloration, Split half reliability (Gutmann's $\lambda 6$ ) and McDonald's $\omega$, Cronbach alpha. The validity contained of face content validity, construct validity through exploratory factorial analysis and confirmatory factor analysis. All analysis had been done through Statistical Package of Social Sciences (SPSS), Statistical Package of Social Sciences-Analysis of Moment Structures (SPSS-Amos) and Jeffrey's Amazing Statistics Program (JASP). Results: A total of 209 pharmacists responded. The majority of responders were Saudi $185(88.52 \%)$. The among responders were males [108 $(61.77 \%)$ ] and females [101 $(48.33 \%)$ ]. The three tests had been done with reliability of 31 questions. The completed responders (185) (mean \pm SD) was $3.236 \pm 0.326$ and McDonald's $\omega$, Cronbach alpha and Gutmann's 16 were $0.980,0.980$ and 0.990 , respectively with $\mathrm{Cl} 95 \%(0.975-0.983)$ and inter-item coloration was 0.607 , while the item-total coloration $>0.53$ and McDonald's $\omega$, Cronbach alpha and Gutmann's $\lambda 6$ value if deleted was $>0.97$. By using Exploratory Factor Analysis (EFA), the Kaiser-Meyer-Olkin Measure of sampling adequacy was 0.966 and Bartlett's test of sphericity with approximate chi-square was $<0.001$. The commonalities extraction for all questions was $>0.57$, the related components were four of all 31 questions in four components as suggested. They were not confirmed by confirmatory with statistically significant $(p<0.001)$ of the factor model, by factor analysis, by scree plot and pathway analysis and fit not with the original survey changed to 3 factor loading. The confirmatory factor index was (0.761), Tucker-Lewis Index (TLI) was (0.737), Goodness of fit index (GFI) was (0.844) and expected cross validation index (ECVI) (9.029). The collinearity of 23 questions was autocorrelation $(2.609 e-5)$ with not statistically significant ( $p=0.951$ ). The majority of 23 question had Enjuone value close to number 1 , while 11 questions had condition index more than 30 . All of the 23 questions had The Variance inflation factor (VIF) less than 10 except four questions and had tolerance more than 0.1 except four questions. Conclusion: The reliability and validity related to the corrected survey of biostatistics analysts used in pharmaceutical research in the Kingdom of Saudi Arabia were high. The pharmacy practice was properly used in further future research in Saudi Arabia.

Key words: Reliability, Validity, Biostatical Analysis, Knowledge, Pharmacy, Research, Survey, Saudi Arabia.
\end{abstract}

\section{INTRODUCTION}

Reliability and validity are a common concept used in the pharmacy research field. The reliability was defined as scale or test is reliable to the extent that repeat measurements made by it under constant conditions will give the same result" ${ }^{\prime \prime}$ that's you need tools to assure to give the same results once repeated the test. The reliability consisted of various types that are including test-retest reliability or, parallel from reliability and inter-rater reliability, or internal consistency reliability. The internal reliability needs various biostatistical analysis to implement that including Cronbach alpha and Machdolan W. The validity was defined as "the degree to which the researcher has measured what he has set out to measure". The validation had several types with common which were content and construct validity. The content validity easy to use, while contract validity needs biostatistical analysis with principal component and factorial analysis. The validity had several advantages that's including unified the goal of the project; it is part of quality management processes, to keep the question of the survey within one field and target. The construct validity needs special education and training to implement through software biostatistical programs.

In the past years, the pharmacy strategic plan involved the pharmacy research and validated the role of the pharmacists with a clinical and economic impact in the pharmacy practice. ${ }^{1-5}$ Besides, pharmacy indicators of follow-up and a successful pharmacy strategic plan were implemented, ${ }^{1}$ including patients and pharmacist satisfaction. As a result, various publications related to the pharmacist with outcome in practice revealed. Also, multiple cross-sections study through a self-survey about patient satisfaction with pharmacy services and pharmacist job satisfaction also revealed. ${ }^{6}$ Both surveys extracted from previous literature with a new design appropriate for pharmacy practice in the Kingdom of Saudi Arabia. Even though both studies were very edifying, but reliability and validity are highly suggested for both surveys. Some studies in the biostatistical knowledge of the healthcare 
professional including pharmacist; they had been done with a very brief discussion about reliability and validity or eve not existed. ${ }^{7-11}$ Another study done in pharmacy research knowledge of biostatistics with reliability checked and without detail information about the reliability or validity of the study. ${ }^{12}$ The authors were not familiar with an investigation about the reliability or validity of the biostatistics analysis knowledge in Saudi Arabia. The aim of the current study is to declare the reliability and validity of the biostatistics analysis of a pharmacy research survey in the Kingdom of Saudi Arabia.

\section{METHODS}

\section{Survey Development}

It is a cross-section survey developed by the authors and hit the research team. It's based on the updated literature and national and international accreditation standards organizations. ${ }^{13-16}$ The survey contained two parts: the first section about demographic data for responders including genders, nationality with dichotomous data and age with ordinal data. The rest of the data uses as ordinal information, including the responder's qualifications, responders background, education, the board of pharmaceutical certificate and the current job and experience. The other section of demographic information was hospital data with ordinal data and included hospital bed capacity based on the ministry of health classification; the university updated hospital accreditation status from national and international accreditation institutions. The second part of the survey about patient satisfaction of pharmacy services. The section divided into several domains and each domain had several questions related to the domain. The answers of the domains were likely with 1 (I do not need this knowledge), 2 (I do not have knowledge), 3 (Weak knowledge), 4 (Incomplete knowledge) and 5 (Complete knowledge). A pilot study was done through the authors and the team distributes electronically or manually to target responders $20-30$ as a pilot. Sometimes they interview patients to assure all the questions clear and understood by the responders. All comments brought for discussion. The correction of the survey had been done based on the agreement of most research members. ${ }^{17}$ The research team tested the McDonald's $\omega$ and Cronbach alpha for internal reliability in the pilot responders by using Statistical Package of Social Sciences (SPSS), Statistical Package of Social Sciences-Analysis of Moment Structures (SPSS-Amos) and Jeffrey's Amazing Statistics Program (JASP). ${ }^{18}$

\section{Internal Consistency Reliability}

\section{Item-Item Correlation}

The method used to measure each question to another one, with high coloration results in more than 0.7 that is high internal consistency reliability survey. ${ }^{18,19}$

\section{Item-Total Correlation}

The method to measure the total questions correlates with each question alone. The high results more than 0.7 , the high correlate internal consistency reliability of the survey. ${ }^{18,19}$

\section{Split Half Reliability (Gutmann's $\lambda 6$ )}

The method used through the SPSS and JASP program with the scale option and reliability section. All questions to scale or ordinal data need to be measured. The test used a split-half option. The SPSS or JASP will split the question into two half and measures the coloration of the two groups. The high results more 0.9 about coloration means higher reliability with internal consistency. ${ }^{17,20,21}$
McDonald's $\omega$, Cronbach Alpha

The research team applied McDonald's $\omega$ and Cronbach alpha for internal reliability by using SPSS and JASP. All questions with a scale or ordinal data included in the analysis. The scale more than 0.9 ; it will be excellent internal consistency, 0.7-0.9 means good reliability, 0.3-0.6 means not acceptable reliability and the score less than 0.3 means weak reliability. ${ }^{17,22,23}$

\section{Face Content Validity}

The principle authors planned the survey and team research revised independently. Each member revised all survey content questions based on the updated literature and experience. Any violations had been sent to all research team for further discussion and agreements. The survey had been corrected and agreement from the research team. One of the team members transferred all surveys to the Arabic language and double-checked by all team members again for content and accurate translation. ${ }^{17,19}$

\section{Construct Validity \\ Exploratory Factorial Analysis}

The method was used for the construct validity of the survey. The factor used univariate description and Kaiser-Myer-Olin measure of sampling adequacy and Bartlett's test sphericity. The extraction was used principal components analysis, the Eigen values greater than 1 with the maximum iteration of convergence 25 and display through un-rotated faction solution and scree plot. The rotation used Varimax. ${ }^{15,21}$

\section{Confirmatory Factor Analysis}

The test was done through SPSS-AMOS and JASP software programs with factor variances, R-Sequated, fit measurements, factor loading, without emulation, error calculated with CI 95\% and robust method, it was with the auto-estimator and without standardization, it was with pathway analysis. ${ }^{15,21}$

\section{Collinearity}

The test was done through JASP with linear regression for collinearity diagnostics including Eigen value and condition index, the coefficient used with CI 95\% tolerance and variance inflation factor, the model fit through ANOVA and auto-correlation with Durbin-Watson. ${ }^{24}$

\section{Statically Analysis}

Various biostatical analysis was done in the current study like the McDonald's $\omega$, Cronbach alpha and Gutmann's $\lambda 6$ for calculation reliability. The Kaiser-Meyer-Olkin measure of sampling adequacy and Bartlett's test of Sphericity with approximate chi-square for Exploratory Factor Analysis (EFA) was used during the study. The factor variances, R-Sequated, fit measurements, factor loading, without emulation, error calculated with CI 95\% and robust method, it was with the auto-estimator and without standardization, it was with scree plot and pathway analysis. Collinearity had been diagnostician through linear regression, the variance inflation factor was calculated, the model fit through ANOVA and auto-correlation with Durbin-Watson. All biostatistical analysis was done by the Statistical Package of Social Sciences (SPSS), SPSS-AMOS and Jeffrey's Amazing Statistics Program (JASP).

\section{RESULTS}

A total of 209 pharmacists responded. The majority of responders were Saudi 185 (88.52\%). The among responders were males 108 (61.77\%) and females $101(48.33 \%)$. Most of the responders were in the age (18$29)$ years and age (30-44) years were $104(49.67 \%)$ and $78(37.32 \%)$, respectively. The majority of responders had a doctor of pharmacy and a Bachelor's degree in pharmacy was 92 (44.32\%) and 81 (38.94\%), respec- 


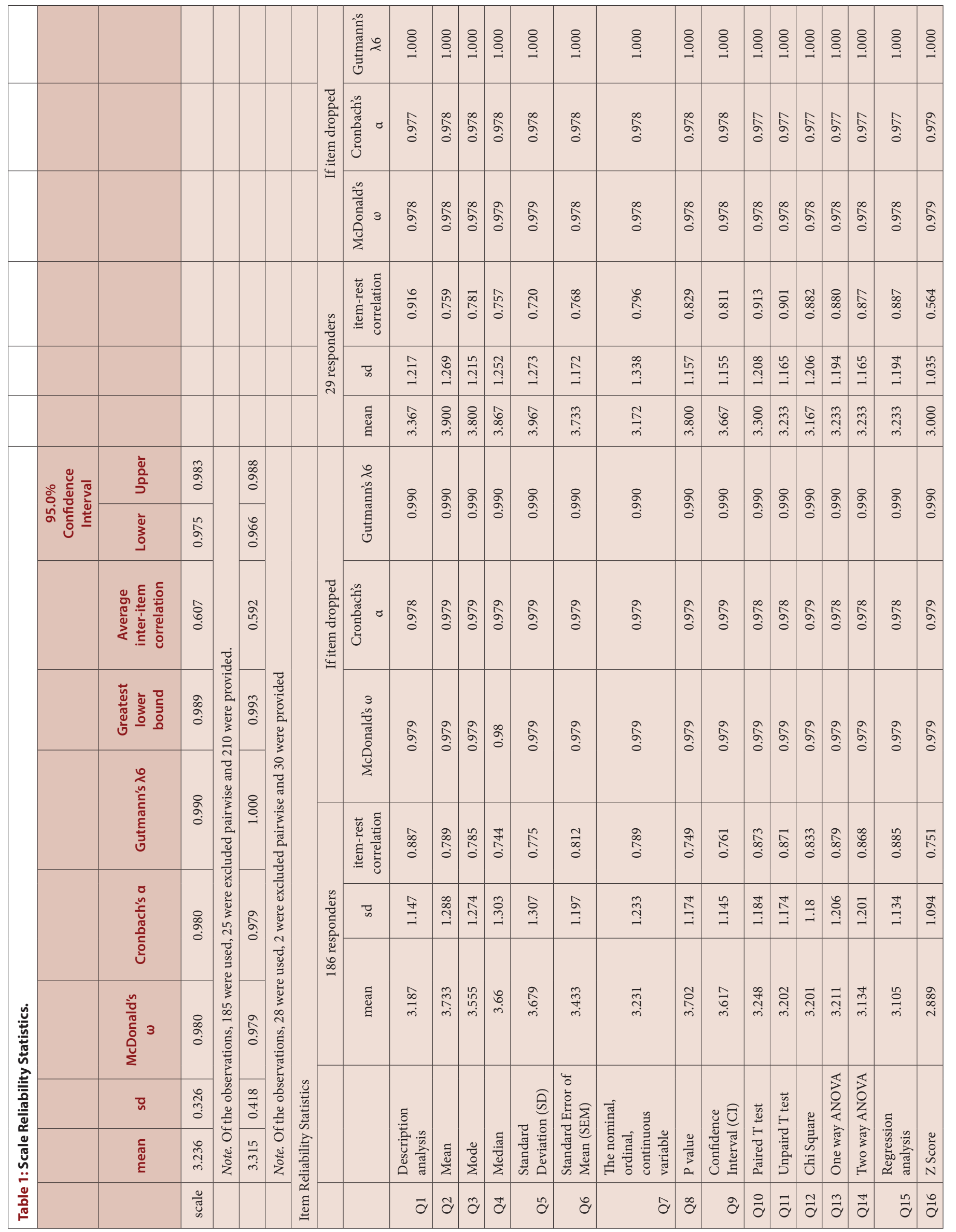




\begin{tabular}{|c|c|c|c|c|c|c|c|c|c|c|c|c|c|c|}
\hline$\underset{-}{\stackrel{-}{8}}$ & $\underset{-}{8}$ & 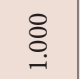 & $\underset{-}{\stackrel{-}{8}}$ & 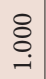 & 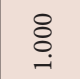 & $\underset{-}{\stackrel{8}{8}}$ & 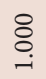 & 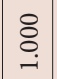 & $\underset{\text { ¿ }}{\stackrel{8}{8}}$ & $\underset{-}{\stackrel{-}{0}}$ & 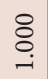 & 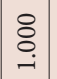 & 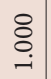 & 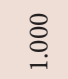 \\
\hline 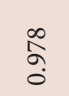 & $\begin{array}{l}\infty \\
\hat{a} \\
0\end{array}$ & 命 & $\begin{array}{l}\infty \\
\hat{\sigma} \\
0\end{array}$ & $\begin{array}{l}\hat{a} \\
\hat{o}\end{array}$ & $\begin{array}{l}\infty \\
\hat{\sigma} \\
0\end{array}$ & $\begin{array}{l}\infty \\
\hat{a} \\
0\end{array}$ & $\begin{array}{l}\infty \\
\hat{\sigma} \\
\vdots\end{array}$ & $\begin{array}{l}\infty \\
\hat{a} \\
0\end{array}$ & مิ & $\begin{array}{l}\hat{\jmath} \\
\hat{\sigma}\end{array}$ & ลิ & $\begin{array}{l}\hat{a} \\
\hat{a}\end{array}$ & 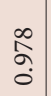 & 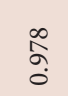 \\
\hline $\begin{array}{l}\text { నิ } \\
\text { o. }\end{array}$ & $\begin{array}{l}\widehat{a} \\
\hat{o}\end{array}$ & $\begin{array}{l}\infty \\
\hat{\sigma} \\
0\end{array}$ & $\begin{array}{l}\infty \\
\hat{a} \\
0\end{array}$ & 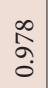 & $\begin{array}{l}\infty \\
\hat{a} \\
0\end{array}$ & $\begin{array}{l}\infty \\
\hat{a} \\
0\end{array}$ & $\begin{array}{l}\hat{\sigma} \\
\text { ડે }\end{array}$ & $\begin{array}{l}\hat{a} \\
\hat{\sigma}\end{array}$ & $\begin{array}{l}\stackrel{2}{\alpha} \\
\hat{\sigma}\end{array}$ & 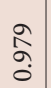 & $\begin{array}{l}\stackrel{2}{0} \\
\stackrel{\circ}{0}\end{array}$ & $\begin{array}{l}\infty \\
\hat{\sigma} \\
0\end{array}$ & $\begin{array}{l}\hat{\sigma} \\
\text { ลे }\end{array}$ & 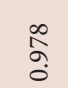 \\
\hline $\begin{array}{l}\text { \&̊ } \\
\text { ơ }\end{array}$ & $\underset{\mathfrak{N}}{\stackrel{N}{0}}$ & 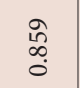 & $\begin{array}{l}N \\
\stackrel{N}{\hat{0}} \\
0\end{array}$ & $\begin{array}{l}0 \\
\stackrel{0}{0} \\
\infty \\
0\end{array}$ & $\begin{array}{l}\overrightarrow{\widehat{\Omega}} \\
0\end{array}$ & $\begin{array}{l}\stackrel{1}{n} \\
\stackrel{0}{0}\end{array}$ & $\begin{array}{l}n \\
0 \\
0 \\
0\end{array}$ & $\begin{array}{l}n \\
\hat{\imath} \\
0 \\
0\end{array}$ & $\begin{array}{l}0 \\
1 n \\
0\end{array}$ & $\begin{array}{l}\overrightarrow{\vec{n}} \\
0 \\
0\end{array}$ & $\begin{array}{l}0 \\
\text { in } \\
0 \\
0\end{array}$ & $\begin{array}{l}0 \\
0 \\
0 \\
0 \\
0\end{array}$ & 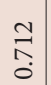 & $\begin{array}{l}\infty \\
\stackrel{0}{0} \\
0\end{array}$ \\
\hline$\underset{\sim}{\stackrel{M}{\rightarrow}}$ & 12. & $\stackrel{\infty}{=}$ & $\begin{array}{l}\mathscr{0} \\
\stackrel{0}{\circ} \\
\stackrel{-}{2}\end{array}$ & $\underset{I}{\stackrel{I}{I}}$ & 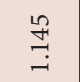 & 㫄 & $\stackrel{\circ}{\stackrel{8}{\circ}}$ & 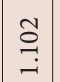 & 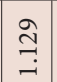 & $\begin{array}{l}\stackrel{2}{2} \\
\stackrel{2}{0}\end{array}$ & تِ & 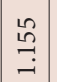 & $\begin{array}{l}\infty \\
\infty \\
0 \\
0\end{array}$ & $\begin{array}{l}\infty \\
\stackrel{\infty}{\infty} \\
\stackrel{-}{0}\end{array}$ \\
\hline $\begin{array}{l}\hat{\otimes} \\
\dot{m}\end{array}$ & $\begin{array}{l}\hat{0} \\
\text { m} \\
\dot{m}\end{array}$ & $\begin{array}{c}m \\
\tilde{o} \\
\dot{m}\end{array}$ & $\begin{array}{c}\tilde{N} \\
\infty \\
i\end{array}$ & $\underset{\tilde{n}}{\tilde{N}}$ & $\begin{array}{l}\hat{\alpha} \\
\stackrel{\text { N }}{\text { in }}\end{array}$ & $\begin{array}{l}\hat{D} \\
\infty \\
i\end{array}$ & $\begin{array}{c}\stackrel{m}{\hat{n}} \\
\stackrel{n}{N}\end{array}$ & $\begin{array}{l}\stackrel{8}{8} \\
\stackrel{0}{i}\end{array}$ & $\begin{array}{l}m \\
\hat{b} \\
\dot{m}\end{array}$ & $\underset{\dot{f}}{\hat{\sigma}}$ & $\begin{array}{l}\hat{\circ} \\
i n \\
m\end{array}$ & $\begin{array}{l}\stackrel{8}{0} \\
\stackrel{0}{m}\end{array}$ & $\begin{array}{l}\stackrel{8}{\stackrel{2}{c}} \\
\text { i }\end{array}$ & $\begin{array}{l}\text { ¿ } \\
\text { mे }\end{array}$ \\
\hline ภू & ๙ু & ஃ̊ & $\begin{array}{l}\text { ̊ू } \\
\text { o }\end{array}$ & ๙ু & ڤু & $\begin{array}{l}\text { ڤू } \\
\text { o. }\end{array}$ & $\begin{array}{l}\stackrel{2}{\circ} \\
\text { o }\end{array}$ & $\begin{array}{l}\text { ஃ. } \\
\vdots\end{array}$ & ڤू & $\vec{a}$ & ஃ. & $\begin{array}{l}2 \\
\swarrow \\
0\end{array}$ & ஓু & बू. \\
\hline ลิ & ลิ & ڤ్̀ & જે & ลิ & ลิ & $\begin{array}{l}\hat{\jmath} \\
\text { مิ }\end{array}$ & $\begin{array}{l}\hat{\alpha} \\
\text { à }\end{array}$ & مิ & $\begin{array}{l}\hat{2} \\
\text { ă }\end{array}$ & 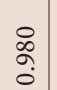 & 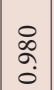 & $\begin{array}{l}\hat{\hat{\alpha}} \\
\hat{\sigma}\end{array}$ & $\begin{array}{l}\hat{\jmath} \\
\text { ă }\end{array}$ & $\begin{array}{l}\stackrel{0}{0} \\
\stackrel{0}{0}\end{array}$ \\
\hline $\begin{array}{l}\hat{a} \\
\text { ò }\end{array}$ & $\stackrel{\infty}{\circ}$ & $\begin{array}{l}\hat{\alpha} \\
\text { à }\end{array}$ & $\begin{array}{l}\hat{\Omega} \\
\hat{\sigma}\end{array}$ & ลิ̊ & ลิ & ลิ & $\begin{array}{l}\hat{a} \\
\text { ลे }\end{array}$ & $\stackrel{\infty}{\circ}$ & $\begin{array}{l}\infty \\
\stackrel{0}{0}\end{array}$ & $\stackrel{\infty}{\circ}$ & $\stackrel{\infty}{\circ}$ & $\stackrel{\infty}{\circ}$ & 吕 & $\stackrel{\infty}{\circ}$ \\
\hline 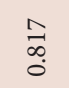 & 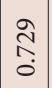 & $\begin{array}{l}\stackrel{1}{0} \\
\infty \\
0 \\
0\end{array}$ & $\begin{array}{c}0 \\
\stackrel{1}{0} \\
0 \\
0\end{array}$ & $\begin{array}{l}n \\
\infty \\
\infty \\
0\end{array}$ & 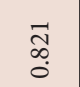 & $\begin{array}{l}\vec{n} \\
\hat{0}\end{array}$ & 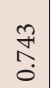 & $\hat{\circ}$ & 命 & $\begin{array}{l}0 \\
\tilde{n} \\
\tilde{n} \\
0\end{array}$ & กี & $\begin{array}{l}\stackrel{+}{+} \\
\stackrel{5}{0}\end{array}$ & $\begin{array}{l}\hat{\infty} \\
\dot{0} \\
0\end{array}$ & in \\
\hline ત્స & $\stackrel{\text { m }}{=}$ & $\stackrel{m}{\longrightarrow}$ & 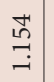 & تُ & $\stackrel{\infty}{\rightrightarrows}$ & 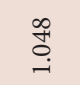 & $\underset{\widetilde{\Xi}}{\widetilde{i}}$ & $\hat{\sigma}$ & ه̊ & 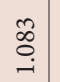 & $\stackrel{\infty}{\stackrel{\overbrace{}}{\leftrightarrows}}$ & $\begin{array}{c}\stackrel{0}{o} \\
\stackrel{-}{-}\end{array}$ & $\begin{array}{l}\vec{\infty} \\
\stackrel{0}{0}\end{array}$ & $\stackrel{\infty}{\leftrightarrows}$ \\
\hline ले & 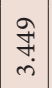 & 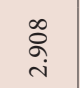 & $\begin{array}{l}\overrightarrow{\widehat{\alpha}} \\
\mathrm{i}\end{array}$ & ڤૂ & $\underset{\widetilde{\Lambda}}{\stackrel{\text { N }}{ }}$ & $\stackrel{\leftrightarrow}{\infty}$ & $\widehat{\grave{i}}$ & $\begin{array}{l}\hat{6} \\
\hat{i} \\
i\end{array}$ & $\begin{array}{c}\infty \\
\infty \\
\infty \\
n \\
n\end{array}$ & $\begin{array}{l}\stackrel{\vec{\omega}}{\infty} \\
\dot{m}\end{array}$ & ஸ़' & $\mid \begin{array}{c}\hat{\alpha} \\
\dot{\infty} \\
\dot{m}\end{array}$ & $\underset{\mathbb{Z}}{\stackrel{\text { S }}{i}}$ & $\begin{array}{l}\stackrel{\leftrightarrow}{0} \\
\stackrel{m}{m}\end{array}$ \\
\hline 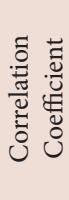 & 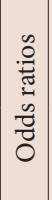 & 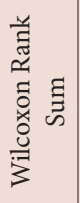 & 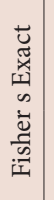 & 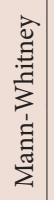 & 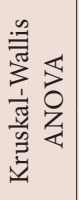 & 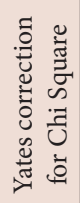 & 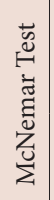 & 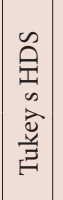 & 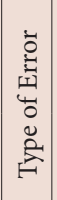 & 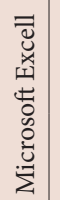 & 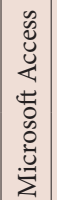 & $\begin{array}{l}\tilde{n} \\
\tilde{\omega}\end{array}$ & 岕 & 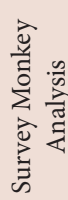 \\
\hline $\bar{\partial}$ & $\stackrel{\infty}{\sigma}$ & $\overrightarrow{\vec{\sigma}}$ & $\stackrel{\tilde{d}}{0}$ & $\overrightarrow{\tilde{d}}$ & $\tilde{d}$ & $\tilde{\tilde{a}}$ & $\stackrel{\ddot{d}}{\tilde{d}}$ & $\stackrel{\mathscr{2}}{\tilde{\sigma}}$ & $\stackrel{\mathscr{Z}}{\mathscr{d}}$ & $\hat{\tilde{\sigma}}$ & $\stackrel{\infty}{\widetilde{\sigma}}$ & $\stackrel{\partial}{\partial}$ & ஜે & $\overrightarrow{\tilde{a}}$ \\
\hline
\end{tabular}

tively. Most of the pharmacists 16 (8\%) had not certified of pharmaceutical specialties 193 (92\%).

\section{Reliability}

The three tests had been done of reliability of 31 questions for the initial (29) responders the (mean \pm SD) was $3.315 \pm 0.418$ and McDonald's $\omega$, Cronbach alpha and Gutmann's $\lambda 6$ were $0.979,0.979$ and 1.000, respectively with CI 95\% (0.966-0.988), while inter-item coloration was 0.592 . After the completed number of responders (185), mean \pm SD was $3.236 \pm$ 0.326 and McDonald's $\omega$, Cronbach alpha and Gutmann's $\lambda 6$ were 0.980 , 0.980 and 0.990 , respectively with CI 95\% (0.975-0.983) and inter-item coloration was 0.607 . Among the 30 responders, all questions item-total coloration $>0.40$ and McDonald's $\omega$, Cronbach alpha and Gutmann's $\lambda 6$ value if deleted was $>0.97$, while with responders' number (185), the item-total coloration $>0.53$ and McDonald's $\omega$, Cronbach alpha and Gutmann's $\lambda 6$ value if deleted was $>0.97$ (Table 1 ). The Split-Half reliability of 185 valid cases and 31 items; the Cornbrash's Alpha of part 1 was 0.973 , while part 2 was 0.952 , the correlation between forms were 0.883 . The Spearman-Brown Coefficient of unequal length was 0.938 and Guttman Split-Half Coefficient was 0.925 (Table 2).

\section{Validity}

By using Exploratory Factor Analysis (EFA), the Kaiser-Meyer-Olkin measure of sampling adequacy was (0.966) and Bartlett's test of Sphericity with approximate chi-square was $<0.001$ (Table 3 ). The commonalities extraction for all questions was $>0.57$, the related components were four of all 31 questions in components 4 as suggested as declared with scree plot (Figure 1). They were not established by confirmatory with statistically significant $(p<0.001)$ of the factor model, by factor and pathway analysis and fit not with the original survey and changed to 3 factor loading. (Figure 2). The square, multiple correlations of the questions

\begin{tabular}{|c|c|c|c|}
\hline \multicolumn{4}{|c|}{ Case Processing Summary } \\
\hline & & $\mathrm{N}$ & $\%$ \\
\hline \multirow{3}{*}{ Cases } & Valid & 185 & 88.1 \\
\hline & Excluded $^{\mathrm{a}}$ & 25 & 11.9 \\
\hline & Total & 210 & 100.0 \\
\hline \multicolumn{4}{|c|}{ a. Listwise deletion based on all variables in the procedure. } \\
\hline \multicolumn{4}{|c|}{ Reliability Statistics } \\
\hline \multirow[t]{5}{*}{ Cronbach's Alpha } & Part 1 & Value & .973 \\
\hline & & $\mathrm{N}$ of Items & $16^{\mathrm{a}}$ \\
\hline & \multirow{2}{*}{ Part 2} & Value & .952 \\
\hline & & $\mathrm{N}$ of Items & $15^{\mathrm{b}}$ \\
\hline & \multicolumn{2}{|c|}{ Total $\mathrm{N}$ of Items } & 31 \\
\hline \multicolumn{3}{|c|}{ Correlation Between Forms } & .883 \\
\hline \multirow{2}{*}{$\begin{array}{l}\text { Spearman-Brown } \\
\text { Coefficient }\end{array}$} & \multicolumn{2}{|c|}{ Equal Length } & .938 \\
\hline & \multicolumn{2}{|c|}{ Unequal Length } & .938 \\
\hline \multicolumn{3}{|c|}{ Guttman Split-Half Coefficient } & .925 \\
\hline \multicolumn{4}{|c|}{$\begin{array}{l}\text { a. The items are: Description analysis, Mean, Mode, Median, Standard Devia- } \\
\text { tion (SD), Standard Error of Mean (SEM), The nominal, ordinal, continuous } \\
\text { variable, P value, Confidence Interval (CI), Paired T test, Unpaird T test, Chi } \\
\text { Square, One way ANOVA, Two way ANOVA, Regression analysis, Z Score. }\end{array}$} \\
\hline \multicolumn{4}{|c|}{$\begin{array}{l}\text { b. The items are: Correlation Coefficient, Odds ratios, Wilcoxon Rank Sum, } \\
\text { Fisher s Exact, Mann-Whitney, Kruskal-Wallis ANOVA, Yates correction for } \\
\text { Chi Square, McNemar Test, Tukey s HDS, Type of Error, Microsoft Excell, Mi- } \\
\text { crosoft Access, SPSS, SAS, Survey Monkey Analysis. }\end{array}$} \\
\hline
\end{tabular}



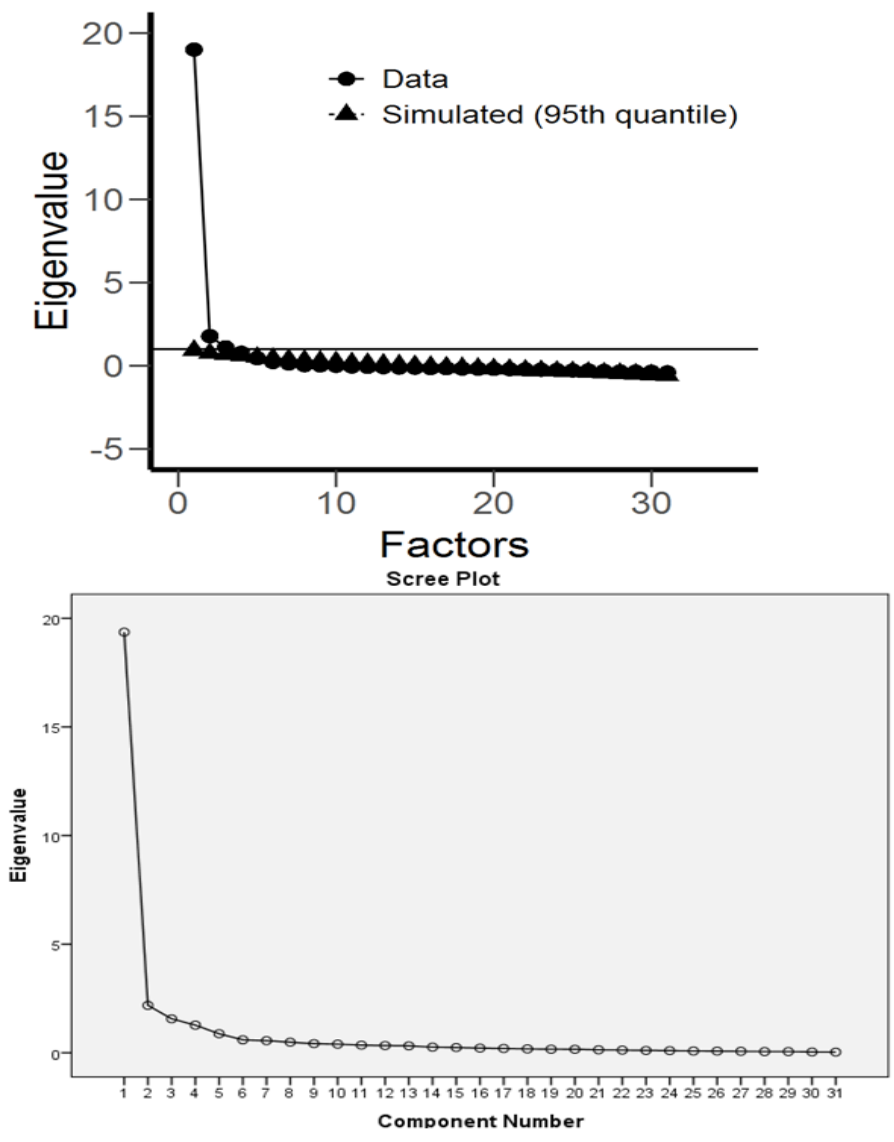

Figure1: Exploratory Factor Analysis Scree Plot
R2 were from (0.450 to 0.911$)$, while factor loading was all the questions $>0.69$ and it was a range $(0.692-1.197)$ with $p<0.001$ after removing the 8 questions confirmed with 3 factor loading. In the pathway analysis, each latent factor and observed coloration with $>0.7$ with $p<0.001$ as discovered in pathway analysis. The confirmatory factor index was 0.761 , Tucker-Lewis Index (TLI) was 0.737, Goodness of fit index (GFI) was 0.844 and Expected cross validation index (ECVI) 9.029. Other results Bentler-Bonett Non-normed Fit Index (NNFI) was 0.737, Bentler-Bonett Normed Fit Index (NFI) was 0.733 , Parsimony Normed Fit Index (PNFI) was 0.666 , Bollen's Relative Fit Index (RFI) was 0.706, Bollen's Incremental Fit Index (IFI) 0.762, Relative Noncentrality Index (RNI) was 0.761, Root mean square error of approximation (RMSEA) was 0.176 and Standardized root mean square residual (SRMR) was 0.407 (Table 4 and 5).

\section{Collinearity}

The correlation coefficients of 23 questions was R2 were (0.943) and RMSE was ( 0.376$)$ with statistically significant $(p<0.001)$, while the autocorrelation was $(2.609 \mathrm{e}-5)$ with not statically significant $(p=0.951)$. The majority of 23 question had Enjuone value had close to number 1, while 11 questions had condition index more than 30 . All 23 of the questions had the Variance inflation factor (VIF) less than 10 except four questions and had tolerance more than 0.1 except for questions (Table 6).

\section{DISCUSSION}

Biostatistical analysis is a crucial part of pharmacy research. The biostatistics sciences were an essential part of the study of Pharm D graduate in the Kingdom of Saudi Arabia. All research pharmacists should have a basic knowledge of biostatistics to prevent any mistakes in the results of the research. As a result, the authors and his colleagues did a study to assess the level of knowledge of basic biostatistics among the pharmacists.

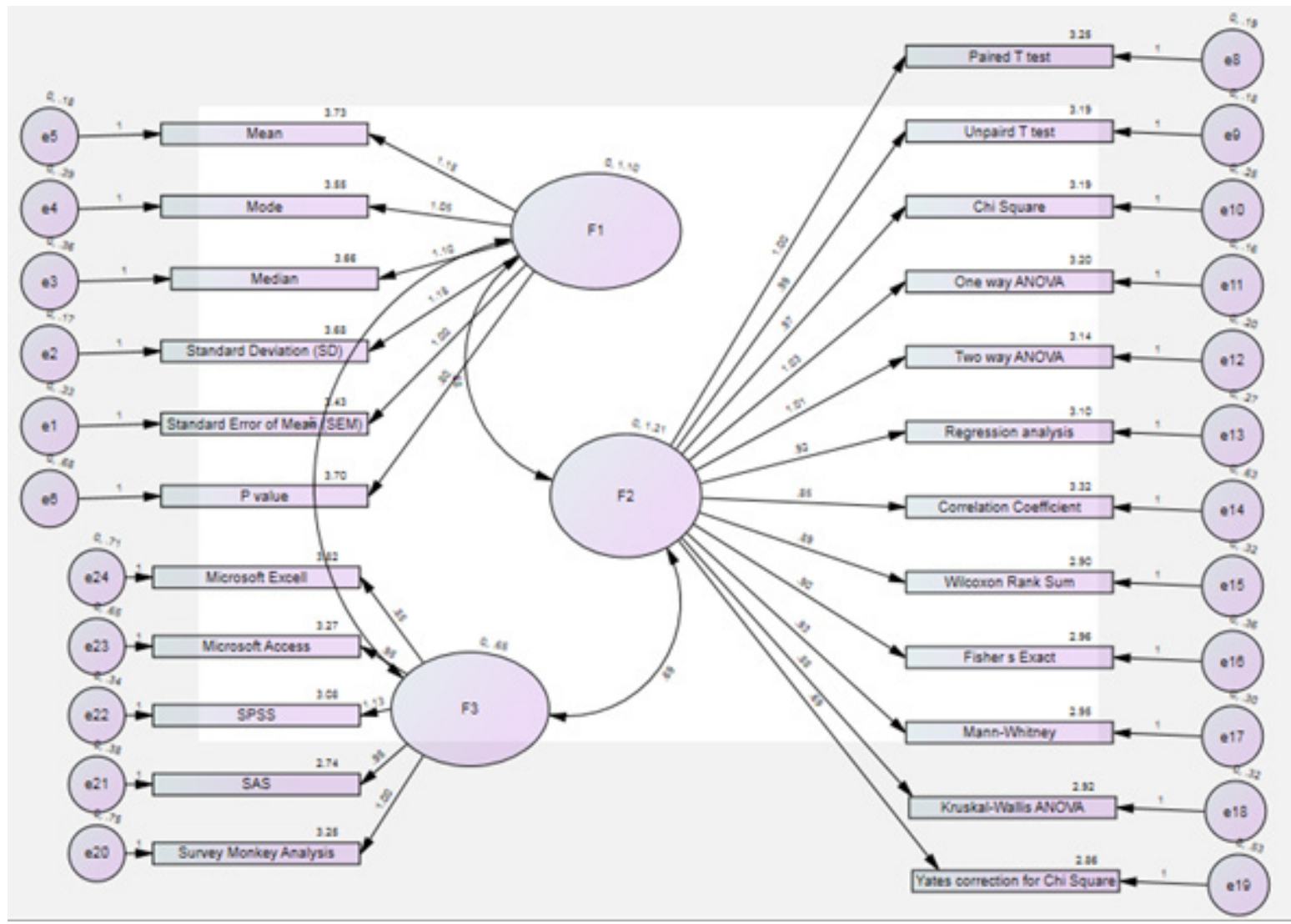

Figure2: confirmatory analysis pathway diagram 


\section{Table 3: Exploratory Factor Analysis Validity.}

\begin{tabular}{|c|c|c|c|c|c|}
\hline & \multicolumn{5}{|c|}{ Exploratory Factor Analysis (EFA) } \\
\hline & \multicolumn{2}{|c|}{$\begin{array}{l}\text { Kaiser-Meyer-Olkin Measure of Sampling Adequacy. } \\
\text { KMO and Bartlett's Test }\end{array}$} & \multirow[b]{2}{*}{ df } & \multirow{2}{*}{$\begin{array}{c}.966 \\
5222.122 \\
\end{array}$} & \\
\hline & Bartlett's Test of Sphericity & Approx. Chi-Square & & & \\
\hline & & & Sig. & 210 & \\
\hline & & & & $<.001$ & \\
\hline & Items & Communalities & & omponent & \\
\hline & & Extraction & Factor 1 & Factor 2 & Factor 3 \\
\hline Q1 & Description analysis & .810 & . & 0.661 & 0.44 \\
\hline Q2 & Mean & .875 & . & 0.635 & 0.447 \\
\hline Q3 & Mode & .809 & . & 0.564 & 0.437 \\
\hline Q4 & Median & .842 & . & 0.598 & . \\
\hline Q5 & Standard Deviation (SD) & .899 & 0.443 & 0.745 & . \\
\hline Q6 & Standard Error of Mean (SEM) & .824 & . & . & 0.822 \\
\hline Q7 & The nominal, ordinal, continuous variable & .707 & . & . & 0.745 \\
\hline Q8 & $P$ value & .764 & 0.408 & . & 0.65 \\
\hline Q9 & Confidence Interval (CI) & .793 & 0.731 & 0.499 & . \\
\hline Q10 & Paired T test & .850 & 0.713 & 0.5 & . \\
\hline Q11 & Unpaird T test & .858 & 0.649 & 0.566 & . \\
\hline Q12 & Chi Square & .830 & 0.708 & 0.513 & . \\
\hline Q13 & One way ANOVA & .872 & . & . & 0.728 \\
\hline Q14 & Two way ANOVA & .846 & . & . & 0.793 \\
\hline Q15 & Regression analysis & .826 & 0.713 & . & . \\
\hline Q16 & Z Score & .624 & 0.43 & 0.763 & . \\
\hline Q17 & Correlation Coefficient & .799 & 0.806 & . & . \\
\hline Q18 & Odds ratios & .772 & 0.831 & . & . \\
\hline Q19 & Wilcoxon Rank Sum & .866 & 0.473 & 0.566 & . \\
\hline Q20 & Fisher s Exact & .828 & 0.441 & 0.765 & . \\
\hline Q21 & Mann-Whitney & .826 & 0.452 & 0.732 & . \\
\hline Q22 & Kruskal-Wallis ANOVA & .849 & 0.445 & 0.713 & . \\
\hline Q23 & Yates correction for Chi Square & .723 & 0.458 & 0.616 & 0.461 \\
\hline Q24 & McNemar Test & .813 & 0.55 & 0.588 & 0.402 \\
\hline Q25 & Tukey s HDS & .805 & 0.556 & . & 0.401 \\
\hline Q26 & Type of Error & .750 & . & 0.426 & 0.798 \\
\hline Q27 & Microsoft Excel & .578 & . & . & 0.469 \\
\hline Q28 & Microsoft Access & .703 & 0.542 & . & 0.435 \\
\hline Q29 & SPSS & .688 & 0.597 & . & 0.475 \\
\hline Q30 & SAS & .683 & 0.674 & . & . \\
\hline Q31 & Survey Monkey Analysis & .669 & 0.403 & . & 0.457 \\
\hline
\end{tabular}


Table 4: The Confirmatory Factor Analysis analysis test.

\begin{tabular}{|c|c|c|c|c|c|c|}
\hline & \multicolumn{6}{|c|}{ Confirmatory Factor Analysis (CFA) } \\
\hline & \multicolumn{2}{|l|}{ Chi-square test } & \multirow[b]{2}{*}{5899.246} & \multirow[b]{2}{*}{253} & \multirow[b]{2}{*}{$P$} & \\
\hline & & Baseline model & & & & \multirow[b]{4}{*}{$p$} \\
\hline & & Factor model & 1577.464 & 230 & $<.001$ & \\
\hline & \multirow[t]{2}{*}{ Items } & \multirow{2}{*}{$\begin{array}{c}\text { Squared Multiple Correlations } \\
\qquad \mathrm{R}^{2} \\
\end{array}$} & \multirow[t]{2}{*}{$\begin{array}{c}\text { Factor } \\
\text { loading (F) }\end{array}$} & \multicolumn{2}{|c|}{ 95\% Confidence Interval } & \\
\hline & & & & Lower & Upper & \\
\hline Q1 & Mean & 0.894 & F1 1.197 & 1.090 & 1.304 & $<.001$ \\
\hline Q2 & Mode & 0.746 & 1.103 & 0.994 & 1.213 & $<.001$ \\
\hline Q3 & Median & 0.784 & 1.157 & 1.054 & 1.259 & $<.001$ \\
\hline Q4 & Standard Deviation (SD) & 0.911 & 1.237 & 1.151 & 1.323 & $<.001$ \\
\hline Q5 & Standard Error of Mean (SEM) & 0.762 & 1.040 & 0.935 & 1.145 & $<.001$ \\
\hline Q6 & $\mathrm{P}$ value & 0.450 & 0.774 & 0.622 & 0.926 & $<.001$ \\
\hline Q7 & Paired T test & 0.849 & F2 1.078 & 0.995 & 1.161 & $<.001$ \\
\hline Q8 & Unpaird T test & 0.849 & 1.067 & 0.981 & 1.153 & $<.001$ \\
\hline Q9 & Chi Square & 0.811 & 1.050 & 0.958 & 1.143 & $<.001$ \\
\hline Q10 & One way ANOVA & 0.876 & 1.116 & 1.037 & 1.195 & $<.001$ \\
\hline Q11 & Two way ANOVA & 0.848 & 1.085 & 0.998 & 1.172 & $<.001$ \\
\hline Q12 & Regression analysis & 0.798 & 1.010 & 0.917 & 1.103 & $<.001$ \\
\hline Q13 & Correlation Coefficient & 0.569 & 0.927 & 0.809 & 1.045 & $<.001$ \\
\hline Q14 & Wilcoxon Rank Sum & 0.771 & 1.002 & 0.896 & 1.108 & $<.001$ \\
\hline Q15 & Fisher s Exact & 0.740 & 0.984 & 0.876 & 1.092 & $<.001$ \\
\hline Q16 & Mann-Whitney & 0.794 & 1.036 & 0.934 & 1.138 & $<.001$ \\
\hline Q17 & Kruskal-Wallis ANOVA & 0.764 & 0.968 & 0.866 & 1.070 & $<.001$ \\
\hline Q18 & Yates correction for Chi Square & 0.545 & 0.770 & 0.642 & 0.897 & $<.001$ \\
\hline Q19 & Microsoft Excel & 0.412 & F3 0.692 & 0.553 & 0.831 & $<.001$ \\
\hline Q20 & Microsoft Access & 0.556 & 0.826 & 0.701 & 0.950 & $<.001$ \\
\hline Q21 & SPSS & 0.664 & 0.877 & 0.779 & 0.975 & $<.001$ \\
\hline Q22 & SAS & 0.567 & 0.741 & 0.628 & 0.853 & $<.001$ \\
\hline Q23 & Survey Monkey Analysis & 0.488 & 0.812 & 0.679 & 0.946 & $<.001$ \\
\hline
\end{tabular}

The study was cross-sectional with a survey that had been distributed to the pharmacist in the Kingdom of Saudi Arabia. The authors did a brief validation of the survey. In the current study, the authors try to evaluate the reliability and validity of the survey. The findings showed a very high reliability of internal consisted of three biostatistical tests either in the pilot responders or completed responders pharmacist and higher than previous medicine residents study. ${ }^{8}$ Besides, the question of the survey had a perfect correlation with each other or among the total questions. The validation of the study had been done through factoring Analysis with expiatory or confirmatory Analysis. The findings of EFA show statically significant of using sample size and suggested four components over 31 questions. The confirmatory Analysis hasn't confined the results of 4 components. It changed to 3 factor loading with statistical significance through a regression model, scree plot and pathway analysis after removing the eight questions. The confirmatory analysts reach up to 0.7 to 0.8 with 23 questions in the survey. The current findings showed that the majority of the questions were collinearity not excised with an emphasis on autocorrelations. If the sample sizes increase, the collinearity might disappear. A unique study was first done in the Middle or Gulf area and all Saudi Arabia. The researcher can esteem the survey and allocate with a good number of sample size.

\section{CONCLUSION}

The knowledge of biostatistical analysis was used in pharmacy research with the corrected survey had high reliability and validation scale level. Further study with a corrected survey in the future with an adequate sample size is suggested in the Kingdom of Saudi Arabia.

\section{ACKNOWLEDGEMENT}

None.

\section{CONFLICT OF INTEREST}

None.

\section{ABBREVIATIONS}

KSA: Kingdom of Saudi Arabia; CFI: Comparative Fit Index; TLI: Tucker-Lewis Index; NNFI: Bentler-Bonett Non-normed Fit Index; NFI: Bentler-Bonett Normed Fit Index; PNFI: Parsimony Normed Fit Index; RFI: Bollen's Relative Fit Index; IFI: Bollen's Incremental Fit In- 


\begin{tabular}{|l|c|c|}
\hline \multicolumn{1}{|c|}{ Table 5: The validity analysis test. } & Value & normal value \\
\hline \multicolumn{1}{|c|}{ Index } & 0.761 & $>0.9$ \\
\hline Comparative Fit Index (CFI) & 0.737 & $>0.9$ \\
\hline Tucker-Lewis Index (TLI) & 0.737 & $>0.9$ \\
\hline Bentler-Bonett Non-normed Fit Index (NNFI) & 0.733 & $>0.9$ \\
\hline Bentler-Bonett Normed Fit Index (NFI) & 0.666 & $>0.9$ \\
\hline Parsimony Normed Fit Index (PNFI) & 0.706 & $>0.9$ \\
\hline Bollen's Relative Fit Index (RFI) & 0.762 & $>0.9$ \\
\hline Bollen's Incremental Fit Index (IFI) & 0.761 & $>0 r=0.08$ \\
\hline Relative Noncentrality Index (RNI) & 0.176 & $>0.04$ \\
\hline & CI $90 \%(0.167-0.184)$ & \\
\hline Root mean square error of approximation (RMSEA) & $\mathrm{P}<0.001$ & \\
\hline Standardized root mean square residual (SRMR) & 0.407 & $>0.9$ \\
\hline Hoelter's critical N $(\alpha=.05)$ & 33.084 & \\
\hline Hoelter's critical N $(\alpha=.01)$ & 35.064 & \\
\hline Goodness of fit index $(\mathrm{GFI)}$ & 0.844 & \\
\hline McDonald fit index $($ MFI) & 0.029 & \\
\hline Expected cross validation index $($ ECVI) & 9.029 & \\
\hline
\end{tabular}

\begin{tabular}{|c|c|c|c|c|c|c|c|c|c|c|c|}
\hline \multicolumn{6}{|c|}{ Collinearity } & \multirow[b]{2}{*}{+} & \multirow[b]{2}{*}{2} & \multicolumn{2}{|c|}{$95 \% \mathrm{Cl}$} & \multirow[b]{2}{*}{$\begin{array}{l}\stackrel{\Xi}{\frac{1}{0}} \\
\frac{\check{0}}{0} \\
\frac{0}{0}\end{array}$} & \multirow[b]{2}{*}{$\stackrel{\Perp}{\supset}$} \\
\hline & 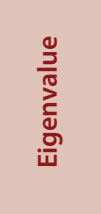 & 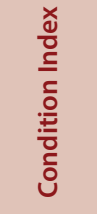 & 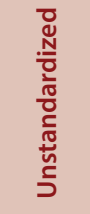 & 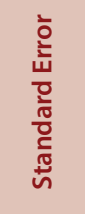 & 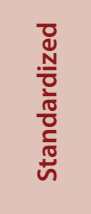 & & & 히 & 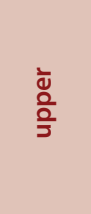 & & \\
\hline Q1 & 22.056 & 1.000 & 0.012 & 0.067 & 0.047 & 0.180 & 0.857 & -0.119 & 0.143 & 0.104 & 9.580 \\
\hline Q2 & 0.224 & 9.933 & 0.018 & 0.056 & 0.074 & 0.332 & 0.741 & -0.091 & 0.128 & 0.147 & 6.814 \\
\hline Q3 & 0.163 & 11.635 & 0.006 & 0.055 & 0.023 & 0.100 & 0.920 & -0.103 & 0.114 & 0.142 & 7.021 \\
\hline Q4 & 0.098 & 14.966 & -0.038 & 0.065 & -0.155 & -0.583 & 0.561 & -0.167 & 0.091 & 0.103 & 9.702 \\
\hline Q5 & 0.064 & 18.625 & 0.072 & 0.059 & 0.269 & 1.222 & 0.223 & -0.044 & 0.189 & 0.149 & 6.691 \\
\hline Q6 & 0.055 & 20.024 & 0.075 & 0.035 & 0.272 & 2.133 & 0.034 & 0.006 & 0.145 & 0.415 & 2.412 \\
\hline Q7 & 0.047 & 21.665 & -0.289 & 0.088 & -1.057 & -3.301 & 0.001 & -0.462 & -0.116 & 0.070 & 14.306 \\
\hline Q8 & 0.039 & 23.784 & 0.110 & 0.090 & 0.397 & 1.219 & 0.225 & -0.068 & 0.287 & 0.068 & 14.618 \\
\hline Q9 & 0.038 & 24.118 & 0.091 & 0.060 & 0.332 & 1.513 & 0.132 & -0.028 & 0.210 & 0.149 & 6.694 \\
\hline Q10 & 0.032 & 26.270 & -0.080 & 0.090 & -0.298 & -0.889 & 0.375 & -0.257 & 0.097 & 0.065 & 15.467 \\
\hline Q11 & 0.028 & 28.084 & 0.107 & 0.082 & 0.393 & 1.310 & 0.192 & -0.054 & 0.268 & 0.080 & 12.449 \\
\hline Q12 & 0.025 & 29.841 & -0.043 & 0.057 & -0.151 & -0.753 & 0.453 & -0.155 & 0.069 & 0.179 & 5.581 \\
\hline Q13 & 0.020 & 32.817 & 0.069 & 0.052 & 0.263 & 1.320 & 0.189 & -0.034 & 0.171 & 0.182 & 5.495 \\
\hline Q14 & 0.020 & 33.176 & 0.001 & 0.075 & 0.004 & 0.016 & 0.987 & -0.148 & 0.150 & 0.100 & 9.958 \\
\hline Q15 & 0.016 & 36.950 & -0.043 & 0.065 & -0.154 & -0.668 & 0.505 & -0.171 & 0.084 & 0.136 & 7.364 \\
\hline Q16 & 0.016 & 37.510 & 0.018 & 0.069 & 0.067 & 0.266 & 0.790 & -0.118 & 0.155 & 0.114 & 8.790 \\
\hline Q17 & 0.014 & 39.127 & 0.017 & 0.066 & 0.058 & 0.254 & 0.800 & -0.113 & 0.147 & 0.137 & 7.287 \\
\hline Q18 & 0.011 & 44.518 & 0.058 & 0.049 & 0.189 & 1.178 & 0.240 & -0.039 & 0.155 & 0.281 & 3.554 \\
\hline
\end{tabular}


Alomi and Rayah.: Reliability and Validity of Biostatical Analysis for Pharmacy Research in Saudi Arabia

\begin{tabular}{|c|c|c|c|c|c|c|c|c|c|c|c|}
\hline Q19 & 0.009 & 48.718 & 0.108 & 0.035 & 0.363 & 3.096 & 0.002 & 0.039 & 0.176 & 0.457 & 2.188 \\
\hline Q20 & 0.009 & 50.525 & 0.038 & 0.039 & 0.131 & 0.980 & 0.329 & -0.038 & 0.114 & 0.403 & 2.480 \\
\hline Q21 & 0.007 & 56.983 & -0.095 & 0.045 & -0.321 & -2.126 & 0.035 & -0.184 & -0.007 & 0.317 & 3.151 \\
\hline Q22 & 0.005 & 69.562 & 0.026 & 0.047 & 0.079 & 0.549 & 0.584 & -0.067 & 0.119 & 0.344 & 2.909 \\
\hline Q23 & 0.004 & 72.552 & 0.064 & 0.033 & 0.234 & 1.945 & 0.054 & $-9.805 e-4$ & 0.130 & 0.500 & 2.001 \\
\hline $\mathrm{R}$ & $\mathrm{R}^{2}$ & Adjusted $\mathrm{R}^{2}$ & RMSE & $\begin{array}{c}\mathrm{R}^{2} \\
\text { Change }\end{array}$ & F Change & df1 & $\mathrm{df} 2$ & $\mathrm{p}$ & & & \\
\hline 0.958 & 0.953 & 0.908 & 0.376 & 0.908 & 71.456 & 32 & 167 & $<.001$ & & & \\
\hline ANOVA & & & & & & & & & $\begin{array}{c}\text { Col } \\
\mathrm{Th}\end{array}$ & $\begin{array}{l}\text { arity } \\
\text { oold }\end{array}$ & \\
\hline Model & Sum of Squares & $\mathrm{df}$ & Mean Square & $\mathrm{F}$ & $\mathrm{p}$ & & $\begin{array}{r}\text { Varian } \\
\text { fact }\end{array}$ & $\begin{array}{l}\text { e inflation } \\
\text { r (VIF) }\end{array}$ & & & \\
\hline Regression & 233.294 & 23 & 7.425 & 10.143 & $<.001$ & & & erance & & & \\
\hline Residual & 23.706 & 168 & 0.135 & 0.141 & & & Cond & $\begin{array}{l}\text { ion index } \\
\text { CI) }\end{array}$ & & & \\
\hline \multirow[t]{4}{*}{ Total } & 257.000 & 191 & & & & & \multicolumn{4}{|c|}{$\begin{array}{l}\text { The eigenvalue (coloration matrix); if it is } \\
\text { close to } 0 \text { collinearity is high, if it is close to } \\
1 \text { there is no collinearity in the data }\end{array}$} & \\
\hline & Durbin-Watson & & & & & & & & & & \\
\hline & Autocorrelation & Statistic & $\mathbf{p}$ & & & & & & & & \\
\hline & $\begin{array}{c}2.609 \mathrm{e}-5 \\
0.0 \\
45\end{array}$ & 1.994 & 0.951 & & & & & & & & \\
\hline
\end{tabular}

dex; RNI: Relative Noncentrality Index; RMSEA: Root Mean Square Error of Approximation; SRMR: Standardized Root Mean Square Residual; GFI: Goodness of Fit Index; MFI: McDonald Fit Index; ECVI: Expected Cross Validation Index; SPSS: Statistical Package of Social Sciences: JASP: Jeffrey's Amazing Statistics Program; ANOVA: Analysis of Variance; SPSS-Amos: Statistical Package of Social Sciences-Analysis of Moment Structures.

\section{REFERENCES}

1. Alomi YA, Alghamdi SJ, Alattyh RA, et al. The evaluation of pharmacy strategic plan in past 2013-2016 and forecasting of new vision 2030 at ministry of health in Saudi Arabia. J Pharm Pract Community Med. 2018;4(2):93-101.

2. Alomi YA, Aldosori N, Alhadab M, et al. The outcomes of clinical pharmacist consultation visits at ministry of health hospitals in Saudi Arabia: Medication safety and pharmacy research. J Pharm Pract Community Med. 2017;3(3):168-75.

3. Alomi1 YA, Fallatah AO, Al-Shubaar N, et al. The clinical outcomes of pharmacist interventions in total parenteral nutrition services in Riyadh City, Saudi Arabia. Int J Pharm Heal Sci. 2019;2(2):135-40.

4. Alomi YA, Aldosori N, Alhadab M, et al. The value of clinical pharmacist consultation visits at ministry of health hospitals in Saudi Arabia: Intravenous admixture services and pharmacy total quality management. J Pharm Pract Community Med. 2017;3(3):161-7.

5. Alomi YA, Aldosori N, Alhadab M, et al. Impact of clinical pharmacist consultation visits at ministry of health hospitals in Saudi Arabia: clinical pharmacy services and pharmacy workforce. J Pharm Pract Community Med. 2017;3(3):15460.

6. Alomi YA, Kurdy L, Aljarad Z, et al. Patient satisfaction of pharmaceutical care of primary care centers at ministry of health in Saudi Arabia. J Pharm Pract Community Med. 2016;2(3):79-87.

7. Ferrill MJ, Norton LL, Blalock SJ. Determining the statistical knowledge of pharmacy practitioners: A survey and review of the literature. Am J Pharm Educ. 1999;63(4):371-6.

8. Windish DM, Huot SJ, Green ML. Medicine residents' understanding of the biostatistics and results in the medical literature. J Am Med Assoc. 2007;298(9):1010-22

9. Al-Zahrani SH, Al-Khail BAA. Resident physician's knowledge and attitudes toward biostatistics and research methods concepts. Saudi Med J.
2015;36(10):1236-40.

10. Polychronopoulou A, Eliades T, Taoufik K, et al. Knowledge of European orthodontic postgraduate students on biostatistics. Eur J Orthod. 2011;33(4):434 40

11. Shetty AC, AlRasheed NM, Albwardi SA. Dental professionals' attitude towards biostatistics. Journal of Dentistry and Oral Hygiene. 2015;7(7):113-8.

12. Alomi YA, Altebainawi A, Alabdullatif A. Biostatistical analysis knowledge of Pharmacy Research in Kingdom of Saudi Arabia. In: ISPOR Europe. Copenhagen, Denmark. 2019;22(S3):S779. Available from: https://www.ispor.org/heorresources/presentations-database/presentation/euro2019-3118/93718.

13. Boynton PM, GreenhalghT. Hands-on guide to questionnaire research: Selecting, designing and developing your questionnaire. Br Med J. 2004;328(7451):1312 5 .

14. Artino AR, LaRochelle JS, Dezee KJ, et al. Developing questionnaires for educational research: AMEE Guide No. 87. Med Teach. 2014;36(6):463-74.

15. Mackenzie SB, Podsakoff PM, Podsakoff NP, et al. Construct measurement and validation procedures in MIS and behavioral research: Integrating new and existing techniques. MIS Q. 2011;35(2):293-334

16. Siny T, Colin FR, Abdullah ST. Avoiding failed spinal anesthesia: "Advik technique" A very rare unusual site of ventilator breathing circuit leakage: Beware. Saudi J Anesth. 2017;11(5):80-9

17. Jain S, Dubey S. Designing and validation of questionnaire. Int Dent Med J Adv Res. 2016;2(1):1-3

18. Kimberlin $\mathrm{CL}$, Winterstein AG. Validity and reliability of measurement instruments used in research. Am J Heal Pharm. 2008;65(23):2276-84.

19. Kumar R. Research Methodology a step-by-step guide for beginners. $3^{\text {rd }}$ edition. SAGE Publications Ltd. 2011.

20. Nath S. Best split-half and maximum reliability. IOSR J Res Method Educ. 2013;3(1):1-8

21. Hervás $A$, Guàrdia OJ, Peró $C M$, et al. A structural equation model for analysis of factors associated with the choice of engineering degrees in a technical university. Abstr Appl Anal. 2013.

22. Viladrich C, Angulo-Brunet A, Doval E. A journey around alpha and omega to estimate internal consistency reliability. An Psicol. 2017;33(3):755-82.

23. Deng L, Chan W. Testing the difference between reliability coefficients alpha and omega. Educ Psychol Meas. 2017;77(2):185-203.

24. Dormann CF, Elith J, Bacher $S$, et al. Collinearity: A review of methods to deal with it and a simulation study evaluating their performance. Ecography. 2013:36(1):27-46 